\title{
Improved Subgradient Extragradient Methods for Solving Pseudomonotone Variational Inequalities in Hilbert Spaces
}

\author{
Duong Viet Thong* $\quad$ Phan Tu Vuong ${ }^{\dagger}$
}

\begin{abstract}
The purpose of this work to investigate pseudomonotone and Lipschitz continuous variational inequalities in real Hilbert spaces. For solving this problem, we propose two new methods which combine advantages of the subgradient extragradient method and the projection contraction method. Similar to some recent developments, the proposed methods do not require the knowledge of the Lipschitz constant associated with the variational inequality mapping. Under suitable mild conditions, we establish the weak and strong convergence of the proposed algorithms. Moreover, linear convergence is obtained under strong pseudomonotonicity and Lipschitz continuity assumptions. Numerical examples in fractional programming and optimal control problems demonstrate the potential of our algorithms as well as compare their performances to several related results.
\end{abstract}

Key words: Projection and contraction method; subgradient extragradient method; Mann type method; variational inequality problem; pseudomonotone mapping.

\section{Introduction}

Let $H$ be a real Hilbert space with inner product $\langle\cdot, \cdot\rangle$ and norm $\|\cdot\|$. Let $C$ be a nonempty, closed and convex subset of $H$. Let $A: H \rightarrow H$ be a single-valued continuous mapping. We consider classical variational inequality (VI) in the sense of Fichera [18] and Stampacchia [37] (see also Kinderlehrer and Stampacchia [25]) which is formulated as follows: Find a point $x^{*} \in C$ such that

$$
\left\langle A x^{*}, x-x^{*}\right\rangle \geq 0 \quad \forall x \in C .
$$

We denote by $\Omega$ the solution set of the VI (1), which is assumed to be nonempty.

Variational inequality (VI) is a very general mathematical model with numerous applications in economics, engineering mechanics, transportation, and many more, see for example, [3, 17, 25, 26]. During the last decades, many algorithms for solving VIs have been proposed in the literature, see e.g. $[15,16,17,25]$. Typically, some kinds of monotonicity are needed for proving the convergence of proposed algorithms. While most of the existing methods are applicable to solving monotone VIs, there are only a few methods can be applied to solving pseudomonotone VIs. It is well known that pseudoconvexity of a function can be characterized by pseudomonotonicity of the gradient mapping $[2,20]$. One of the most important applications of pseudoconvex problem is the fractional programming. Indeed, a fractional function is pseudocovex provided that the

${ }^{*}$ Faculty of Economics Mathematics, National Economics University, Hanoi City, Vietnam. Email: thongduongviet@gmail.com

${ }^{\dagger}$ Mathematical Sciences, University of Southampton, UK and Ho Chi Minh City University of Technology and Education, Vietnam. Email: T.V.Phan@soton.ac.uk 
enumerator is convex, the denominator is concave and both of them are positive and differentiable [5]. This motivates researchers to study numerical methods for solving pseudoconvex programming and pseudomonotone VIs.

Korpelevich [27] (also by Antipin [1] independently) introduced the extragradient method for solving pseudomonotone VIs, which requires two projections onto the feasible set in each iteration. One of the important extensions of the extragradient method is the projection and contraction method proposed by He [21] and Sun [38]. This method also consists of two inner steps per iteration but using a different direction which improved significantly the speed of convergence when compared with the extragradient method [6, 21, 38]. Another important extension of the extragradient method is known as the subgradient extragradient method proposed by Censor et al. [11]. In this method, the second projection onto the feasible set is replaced by a projection onto an easy and constructible half-space containing the feasible set. Since the projection onto a half-space is explicit, the subgradient extragradient method significantly reduces the amount of computation comparing to the extragradient method. A combination of these extensions has been recently considered in [14], which takes advantages of both the projection contraction method and the subgradient extragradient method. A drawback of this method is that, to determine stepsizes, it requires line-search procedures containing many additional projections.

The extragradient method and its modifications have been considered for solving VIs in infinite dimensional Hilbert spaces. It was proved that, if the assigned operator is monotone and Lipschitz continuous, then the iterative sequence generated by the extragradient method converges weakly to a solution [23]. Similar results have been also obtained for the subgradient extragradient method $[9,10,40]$ and the projection contraction method $[13,14]$. The extension of these methods to pseudomonotone VIs, however, is not a trivial task. The reason is that, in the monotone setting, one can use the theory of maximal monotone operators to deliver the convergence of the iterative sequence, which is not the case in the pseudomonotone setting. The first attempt in this direction was done in [8], where the authors assumed that the assigned operator is weakly-strongly continuous, i.e., it maps a weakly convergent sequence to a strongly convergence sequence. This assumption is very strong and does not hold even in the simplest case where the assigned operator is the identity one. Recently it has been weakened to a more reasonable weak-weak continuity condition for the extragradient method in $[42,43]$. Similar results for the subgradient extragradient method [42, Remark 3.3] and the forward-backward-forward method [4] were proved by using the same technique. In the convergence analysis of these methods, it is required to know the Lipschitz constant a priori, which is not a simple task. In this paper, motivated by recently active research on pseudomonotone VIs, we propose some new modified schemes of the subgradient extragradient method for solving pseudomonotone and Lipschitz-continuous variational inequalities in real Hilbert spaces. Our schemes have some significant advantages: firstly, no line-search procedure is needed, which reduces the amount of computation in each step. Secondly, they do not require a priori the knowledge of the Lipschitz constant of the associated operator, which is important in practice. Lastly, they are applicable to pseudomonotone VIs, a strictly broader class than monotone VIs $[2,20]$.

After recalling the problem and some basic definitions and results in Section 2, we propose our first scheme and prove the weak convergence of the iterative sequence to a solution of the considered VI in Section 3. As we are working in infinite dimensional Hilbert spaces, the strong convergence is more desirable. Therefore, in Section 4, we modify the first scheme such that the strong convergence can be guaranteed. In Section 5 we present some numerical experiments illustrating the performance of the proposed methods. To demonstrate the pseudomonotonicity, we present numerical results for a class of fractional programming which is pseudoconvex. For the strong convergence illustration, we consider a class of VIs arising in optimal control problem with 
bang-bang control. Final remarks and conclusions are given in Section 6.

\section{Preliminaries}

Let $H$ be a real Hilbert space with inner product $\langle\cdot, \cdot\rangle$ and norm $\|\cdot\|$. The weak convergence of $\left\{x_{n}\right\}$ to $x$ is denoted by $x_{n} \rightarrow x$ as $n \rightarrow \infty$, while the strong convergence of $\left\{x_{n}\right\}$ to $x$ is written as $x_{n} \rightarrow x$ as $n \rightarrow \infty$. For all $x, y \in H$ we have

$$
\|x+y\|^{2} \leq\|x\|^{2}+2\langle y, x+y\rangle
$$

Moreover

$$
\|\alpha x+\beta y+\gamma z\|^{2}=\alpha\|x\|^{2}+\beta\|y\|^{2}+\gamma\|z\|^{2}-\alpha \beta\|x-y\|^{2}-\alpha \gamma\|x-z\|^{2}-\beta \gamma\|y-z\|^{2}
$$

for all $\alpha, \beta, \gamma \in[0,1]$ with $\alpha+\beta+\gamma=1$.

Definition 2.1. ([32, Chapter 9]). Suppose that a sequence $\left\{x_{n}\right\}$ in $H$ converges strongly to $p \in H$. We say that $\left\{x_{n}\right\}$ converges to $p$ with a $Q$-linear rate if there exists $\delta \in(0,1)$ such that,

$$
\limsup _{n \rightarrow \infty} \frac{\left\|x_{n+1}-p\right\|}{\left\|x_{n}-p\right\|}=\delta,
$$

where Q-convergence rate means Quotient-convergence rate. It can define equivalently as follows:

The sequence $\left\{x_{n}\right\}$ in $H$ converges strongly to $p \in H$ with a $Q$-linear rate if there exists $\delta \in(0,1)$ such that,

$$
\left\|x_{n+1}-p\right\| \leq \delta\left\|x_{n}-p\right\| \quad \text { for all sufficiently large } n
$$

Definition 2.2. Let $T: H \rightarrow H$ be an operator. Then

1. $T$ is called L-Lipschitz continuous with constant $L>0$ if

$$
\|T x-T y\| \leq L\|x-y\| \quad \forall x, y \in H,
$$

if $L=1$ then the operator $T$ is called nonexpansive and if $L \in(0,1), T$ is called a contraction.

2. $T$ is called monotone if

$$
\langle T x-T y, x-y\rangle \geq 0 \quad \forall x, y \in H
$$

3. $T$ is called pseudomonotone in the sense of Karamardian [22] if

$$
\langle T x, y-x\rangle \geq 0 \Longrightarrow\langle T y, y-x\rangle \geq 0 \quad \forall x, y \in H
$$

4. $T$ is called $\alpha$-strongly monotone if there exists a constant $\alpha>0$ such that

$$
\langle T x-T y, x-y\rangle \geq \alpha\|x-y\|^{2} \quad \forall x, y \in H ;
$$

5. $T$ is called $\alpha$-strongly pseudomonotone if there exists a constant $\alpha>0$ such that

$$
\langle T x, y-x\rangle \geq 0 \Longrightarrow\langle T y, y-x\rangle \geq \alpha\|x-y\|^{2} \quad \forall x, y \in H
$$

6. The operator $T$ is called sequentially weakly continuous if for each sequence $\left\{x_{n}\right\}$ we have: $x_{n}$ converges weakly to $x$ implies $T x_{n}$ converges weakly to $T x$. 
We note that (6) is only one of the definitions of pseudomonotonicity which can be found in the literature. For every point $x \in H$, there exists a unique nearest point in $C$, denoted by $P_{C} x$ such that $\left\|x-P_{C} x\right\| \leq\|x-y\| \forall y \in C$. $P_{C}$ is called the metric projection of $H$ onto $C$. It is known that $P_{C}$ is nonexpansive. For properties of the metric projection, the interested reader could be referred to Section 3 in [19].

We recall some well known projection methods for solving (pseudo)monotone VIs considered in the literature. The most well known one is extragradient method proposed by Korpelevich [27] (also by Antipin [1] independently). Consider the Euclidean space $\mathbb{R}^{m}$ and let $A: \mathbb{R}^{m} \rightarrow \mathbb{R}^{m}$ be monotone and $L$-Lipschitz continuous operator. The extragradient method has the following form

$$
\left\{\begin{array}{l}
y_{n}=P_{C}\left(x_{n}-\tau_{n} A x_{n}\right), \\
x_{n+1}=P_{C}\left(x_{n}-\tau_{n} A y_{n}\right)
\end{array}\right.
$$

where $\tau_{n} \in(0,1 / L)$ or $\tau_{n}$ is updated by an adaptive rule such that

$$
\tau_{n}\left\|A x_{n}-A y_{n}\right\| \leq \mu\left\|x_{n}-y_{n}\right\|, \quad \mu \in(0,1) .
$$

Observe that the extragradient method requires the evaluation of two orthogonal projections onto $C$ per iteration. The first method which overcomes this obstacle is the projection and contraction method (PC) of He [21] and Sun [38]. For each iteration $n \in \mathbb{N}$ generates point $y_{n}$ in the spirit of (7):

$$
y_{n}=P_{C}\left(x_{n}-\tau_{n} A x_{n}\right),
$$

and then the next iterate $x_{n+1}$ is generated via the following

$$
x_{n+1}=x_{n}-\gamma \eta_{n} d\left(x_{n}, y_{n}\right)
$$

where $\gamma \in(0,2)$,

$$
\eta_{n}:=\frac{\left\langle x_{n}-y_{n}, d\left(x_{n}, y_{n}\right)\right\rangle}{\left\|d\left(x_{n}, y_{n}\right)\right\|^{2}}
$$

and

$$
d\left(x_{n}, y_{n}\right):=x_{n}-y_{n}-\tau_{n}\left(A x_{n}-A y_{n}\right),
$$

with $\tau_{n} \in(0,1 / L)$ or $\tau_{n}$ is updated by some adaptive rule like (8).

The second extension of the extragradient method is known as the subgradient extragradient method proposed by Censor et al. [9, 10, 11]. In this algorithm, the second projection onto the feasible set $C$ is replaced by a projection onto an easy and constructible set which contains $C$. For each $n \in \mathbb{N}$ generate the following sequences,

$$
\left\{\begin{array}{l}
y_{n}=P_{C}\left(x_{n}-\tau A x_{n}\right) \\
T_{n}=\left\{x \in H \mid\left\langle x_{n}-\tau A x_{n}-y_{n}, x-y_{n}\right\rangle \leq 0\right\} \\
x_{n+1}=P_{T_{n}}\left(x_{n}-\tau A y_{n}\right)
\end{array}\right.
$$

where $\tau \in(0,1 / L)$.

Since the projection and contraction and the subgradient extragradient methods require to calculate only one projection onto $C$ per iteration, their computational efforts and performance have an advantage over other existing results in the literature. Recently, [14] introduced a modification of the subgradient extragradient method by using the direction of the projection and contraction method and stepsize rule $\tau_{n}$ satisfying (8). The fact that in order to determine the stepsize $\tau_{n}$, 
[14, Algorithm 3.1] requires a line-search procedure which contains additional projections. At iteration $n$, if this procedure requires many steps to obtain the appropriate $\tau_{n}$ then many projections are needed. On the other hand, [44] proposed two modifications of the subgradient extragradient method without using the projection contraction direction (9) but an adaptive rule which does not require line-search. Observe that the aforementioned methods are applicable to solving monotone Lipschitz VIs. We will propose in this paper some new methods improving the aforementioned methods. To do so, we need to recall the following Lemmas, which are useful for the later convergence analysis.

Lemma 2.1. ([19]) Let $C$ be a nonempty closed convex subset of a real Hilbert space H. Given $x \in H$ and $z \in C$. Then $z=P_{C} x \Longleftrightarrow\langle x-z, z-y\rangle \geq 0 \quad \forall y \in C$. Moreover,

$$
\left\|P_{C} x-P_{C} y\right\|^{2} \leq\left\langle P_{C} x-P_{C} y, x-y\right\rangle \forall x, y \in C .
$$

Lemma 2.2. ([31]) Let $C$ be a nonempty set of $H$ and $\left\{x_{n}\right\}$ be a sequence in $H$ such that the following two conditions hold:

i) for every $x \in C, \lim _{n \rightarrow \infty}\left\|x_{n}-x\right\|$ exists;

ii) every sequential weak cluster point of $\left\{x_{n}\right\}$ is in $C$.

Then $\left\{x_{n}\right\}$ converges weakly to a point in $C$.

Lemma 2.3. ([12]) Consider the problem $V I(C, A)$ with $C$ being a nonempty, closed, convex subset of a real Hilbert space $H$ and $A: C \rightarrow H$ being pseudomonotone and continuous. Then, $x^{*}$ is a solution of $\operatorname{VI}(C, A)$ if and only if

$$
\left\langle A x, x-x^{*}\right\rangle \geq 0 \quad \forall x \in C .
$$

Lemma 2.4. ([35]) Let $\left\{a_{n}\right\}$ be sequence of nonnegative real numbers, $\left\{\alpha_{n}\right\}$ be a sequence of real numbers in $(0,1)$ with $\sum_{n=1}^{\infty} \alpha_{n}=\infty$ and $\left\{b_{n}\right\}$ be a sequence of real numbers. Assume that

$$
a_{n+1} \leq\left(1-\alpha_{n}\right) a_{n}+\alpha_{n} b_{n} \quad \forall n \geq 1 .
$$

If $\lim \sup _{k \rightarrow \infty} b_{n_{k}} \leq 0$ for every subsequence $\left\{a_{n_{k}}\right\}$ of $\left\{a_{n}\right\}$ satisfying $\lim \inf _{k \rightarrow \infty}\left(a_{n_{k}+1}-a_{n_{k}}\right) \geq 0$ then $\lim _{n \rightarrow \infty} a_{n}=0$.

\section{Weak Convergence Analysis}

In this section, we propose modified subgradient extragradient and projection contraction methods for solving VIs.

\section{Algorithm 3.1.}

Initialization: Given $\tau_{0}>0, \mu \in(0,1), \gamma \in(0,2)$. Let $u_{0} \in H$ be arbitrary

Iterative Steps: Given the current iterate $u_{n}$, calculate $u_{n+1}$ as follows:

Step 1. Compute

$$
v_{n}=P_{C}\left(u_{n}-\tau_{n} A u_{n}\right) .
$$

If $u_{n}=v_{n}$ or $A v_{n}=0$ then stop and $v_{n}$ is a solution of $\Omega$. Otherwise 
Step 2. Compute

$$
u_{n+1}=P_{T_{n}}\left(u_{n}-\gamma \tau_{n} \eta_{n} A v_{n}\right),
$$

where

$$
\begin{gathered}
T_{n}=\left\{x \in H \mid\left\langle u_{n}-\tau_{n} A u_{n}-v_{n}, x-v_{n}\right\rangle \leq 0\right\}, \\
\eta_{n}:= \begin{cases}\frac{\left\langle u_{n}-v_{n}, d_{n}\right\rangle}{\left\|d_{n}\right\|^{2}} & \text { if } d_{n} \neq 0, \\
0 & \text { if } d_{n}=0,\end{cases}
\end{gathered}
$$

and

$$
d_{n}:=u_{n}-v_{n}-\tau_{n}\left(A u_{n}-A v_{n}\right) .
$$

Step 3. Update

$$
\tau_{n+1}:=\mu \frac{\left\|u_{n}-v_{n}\right\|}{\left\|A u_{n}-A v_{n}\right\|} \quad \text { if } \quad \tau_{n}\left\|A u_{n}-A v_{n}\right\|>\mu\left\|u_{n}-v_{n}\right\|, \text { otherwise } \tau_{n+1}:=\tau_{n} .
$$

Set $n:=n+1$ and go to Step 1 .

Observe that the projection onto half-space $T_{n}$ in Step 2 is explicit [7, Section 4.1.3, p. 133], therefore, Algorithm 3.1 requires only one projection in Step 1. Moreover, the stepsize $\tau_{n}$ is updated adaptively in Step 3 without requiring the knowledge of the Lipschitz constant $L$. We start the convergence analysis by proving the following Lemma.

Lemma 3.1. Assume that $A$ is L-Lipschitz continuous on $H$. Then the sequence $\left\{\tau_{n}\right\}$ generated by (10) is nonincreasing and

$$
\lim _{n \rightarrow \infty} \tau_{n}=\tau \geq \min \left\{\tau_{0}, \frac{\mu}{L}\right\}
$$

Moreover

$$
\left\|A u_{n}-A v_{n}\right\| \leq \frac{\mu}{\tau_{n+1}}\left\|u_{n}-v_{n}\right\| .
$$

Proof: It is easy to prove this lemma, hence we omit it.

If at some iteration we have $u_{n}=v_{n}$ or $A v_{n}=0$ then Algorithm 3.1 terminates and $v_{n} \in \Omega$. From now on, we assume that $u_{n} \neq v_{n}$ and $A v_{n} \neq 0$ for all $n$.

Lemma 3.2. Assume that $A$ is Lipschitz continuous on $H$ and pseudomonotone on $C$. Then for every $x^{*} \in \Omega$, there exists $n_{0}>0$ such that

$$
\left\|u_{n+1}-x^{*}\right\|^{2} \leq\left\|u_{n}-x^{*}\right\|^{2}-\left\|u_{n}-u_{n+1}-\gamma \eta_{n} d_{n}\right\|^{2}-(2-\gamma) \gamma \frac{\left(1-\frac{\mu \tau_{n}}{\tau_{n+1}}\right)^{2}}{\left(1+\frac{\mu \tau_{n}}{\tau_{n+1}}\right)^{2}}\left\|u_{n}-v_{n}\right\|^{2} \forall n \geq n_{0} .
$$

Proof: Using (11), we have

$$
\begin{aligned}
\left\|d_{n}\right\| & =\left\|u_{n}-v_{n}-\tau_{n}\left(A u_{n}-A v_{n}\right)\right\| \\
& \geq\left\|u_{n}-v_{n}\right\|-\tau_{n}\left\|A u_{n}-A v_{n}\right\| \\
& \geq\left\|u_{n}-v_{n}\right\|-\frac{\mu \tau_{n}}{\tau_{n+1}}\left\|u_{n}-v_{n}\right\| \\
& =\left(1-\frac{\mu \tau_{n}}{\tau_{n+1}}\right)\left\|u_{n}-v_{n}\right\| .
\end{aligned}
$$


Since $\lim _{n \rightarrow \infty}\left(1-\frac{\mu \tau_{n}}{\tau_{n+1}}\right)=1-\mu>0$, there exists $n_{0} \in \mathbb{N}$ such that

$$
1-\frac{\mu \tau_{n}}{\tau_{n+1}}>\frac{1-\mu}{2} \forall n \geq n_{0}
$$

Therefore, for all $n \geq n_{0}$ we get

$$
\left\|d_{n}\right\| \geq \frac{1-\mu}{2}\left\|u_{n}-v_{n}\right\|>0
$$

Since $x^{*} \in \Omega \subset C \subset T_{n}$, using Lemma 2.1 we have

$$
\begin{aligned}
\left\|u_{n+1}-x^{*}\right\|^{2}= & \left\|P_{T_{n}}\left(u_{n}-\gamma \eta_{n} \tau_{n} A v_{n}\right)-P_{T_{n}} x^{*}\right\|^{2} \\
\leq & \left\langle u_{n+1}-x^{*}, u_{n}-\gamma \eta_{n} \tau_{n} A v_{n}-x^{*}\right\rangle \\
= & \frac{1}{2}\left\|u_{n+1}-x^{*}\right\|^{2}+\frac{1}{2}\left\|u_{n}-\gamma \eta_{n} \tau_{n} A v_{n}-x^{*}\right\|^{2}-\frac{1}{2}\left\|u_{n+1}-u_{n}+\gamma \eta_{n} \tau_{n} A v_{n}\right\|^{2} \\
= & \frac{1}{2}\left\|u_{n+1}-x^{*}\right\|^{2}+\frac{1}{2}\left\|u_{n}-x^{*}\right\|^{2}+\frac{1}{2} \gamma^{2} \eta_{n}^{2} \tau_{n}^{2}\left\|A v_{n}\right\|^{2}-\left\langle u_{n}-x^{*}, \gamma \eta_{n} \tau_{n} A v_{n}\right\rangle \\
& -\frac{1}{2}\left\|u_{n+1}-u_{n}\right\|^{2}-\frac{1}{2} \gamma^{2} \eta_{n}^{2} \tau_{n}^{2}\left\|A v_{n}\right\|^{2}-\left\langle u_{n+1}-u_{n}, \gamma \eta_{n} \tau_{n} A v_{n}\right\rangle \\
= & \frac{1}{2}\left\|u_{n+1}-x^{*}\right\|^{2}+\frac{1}{2}\left\|u_{n}-x^{*}\right\|^{2}-\frac{1}{2}\left\|u_{n+1}-u_{n}\right\|^{2}-\left\langle u_{n+1}-x^{*}, \gamma \eta_{n} \tau_{n} A v_{n}\right\rangle .
\end{aligned}
$$

This implies that

$$
\left\|u_{n+1}-x^{*}\right\|^{2} \leq\left\|u_{n}-x^{*}\right\|^{2}-\left\|u_{n+1}-u_{n}\right\|^{2}-2 \gamma \eta_{n} \tau_{n}\left\langle u_{n+1}-x^{*}, A v_{n}\right\rangle .
$$

Since $v_{n} \in C$ and $x^{*} \in \Omega$, we get $\left\langle A x^{*}, v_{n}-x^{*}\right\rangle \geq 0$. By the pseudomonotonicity of $A$, we have $\left\langle A v_{n}, v_{n}-x^{*}\right\rangle \geq 0$, which implies

$$
\left\langle A v_{n}, u_{n+1}-x^{*}\right\rangle \geq\left\langle A v_{n}, u_{n+1}-v_{n}\right\rangle .
$$

Thus, we obtain

$$
-2 \gamma \eta_{n} \tau_{n}\left\langle A v_{n}, u_{n+1}-x^{*}\right\rangle \leq-2 \gamma \eta_{n} \tau_{n}\left\langle A v_{n}, u_{n+1}-v_{n}\right\rangle .
$$

On the other hand, from $u_{n+1} \in T_{n}$ we have

$$
\left\langle u_{n}-\tau_{n} A u_{n}-v_{n}, u_{n+1}-v_{n}\right\rangle \leq 0 .
$$

This implies that

$$
\left\langle u_{n}-v_{n}-\tau_{n}\left(A u_{n}-A v_{n}\right), u_{n+1}-v_{n}\right\rangle \leq \tau_{n}\left\langle A v_{n}, u_{n+1}-v_{n}\right\rangle,
$$

thus

$$
\left\langle d_{n}, u_{n+1}-v_{n}\right\rangle \leq \tau_{n}\left\langle A v_{n}, u_{n+1}-v_{n}\right\rangle
$$

Hence

$$
-2 \gamma \eta_{n} \tau_{n}\left\langle A v_{n}, u_{n+1}-v_{n}\right\rangle \leq-2 \gamma \eta_{n}\left\langle d_{n}, u_{n+1}-v_{n}\right\rangle .
$$

Combining (15) and (16) we get

$$
\begin{aligned}
-2 \gamma \eta_{n} \tau_{n}\left\langle A v_{n}, u_{n+1}-x^{*}\right\rangle & \leq-2 \gamma \eta_{n}\left\langle d_{n}, u_{n+1}-v_{n}\right\rangle \\
& =-2 \gamma \eta_{n}\left\langle d_{n}, u_{n}-v_{n}\right\rangle+2 \gamma \eta_{n}\left\langle d_{n}, u_{n}-u_{n+1}\right\rangle .
\end{aligned}
$$


From (13), we have $d_{n} \neq 0 \quad \forall n \geq n_{0}$, thus $\eta_{n}=\frac{\left\langle u_{n}-v_{n}, d_{n}\right\rangle}{\left\|d_{n}\right\|^{2}}$, which means

$$
\left\langle u_{n}-v_{n}, d_{n}\right\rangle=\eta_{n}\left\|d_{n}\right\|^{2} \quad \forall n \geq n_{0} .
$$

Moreover

$$
\begin{aligned}
2 \gamma \eta_{n}\left\langle d_{n}, u_{n}-u_{n+1}\right\rangle & =2\left\langle\gamma \eta_{n} d_{n}, u_{n}-u_{n+1}\right\rangle \\
& =\left\|u_{n}-u_{n+1}\right\|^{2}+\gamma^{2} \eta_{n}^{2}\left\|d_{n}\right\|^{2}-\left\|u_{n}-u_{n+1}-\gamma \eta_{n} d_{n}\right\|^{2} .
\end{aligned}
$$

Combining (17), (18) and (19) we get for all $n \geq n_{0}$ that

$$
\begin{aligned}
-2 \gamma \eta_{n} \tau_{n}\left\langle A v_{n}, u_{n+1}-x^{*}\right\rangle & \leq-2 \gamma \eta_{n}^{2}\left\|d_{n}\right\|^{2}+\left\|u_{n}-u_{n+1}\right\|^{2}+\gamma^{2} \eta_{n}^{2}\left\|d_{n}\right\|^{2}-\left\|u_{n}-u_{n+1}-\gamma \eta_{n} d_{n}\right\|^{2} \\
& =\left\|u_{n}-u_{n+1}\right\|^{2}-\left\|u_{n}-u_{n+1}-\gamma \eta_{n} d_{n}\right\|^{2}-(2-\gamma) \gamma \eta_{n}^{2}\left\|d_{n}\right\|^{2} .
\end{aligned}
$$

Substituting (20) into (14) we get

$$
\left\|u_{n+1}-x^{*}\right\|^{2} \leq\left\|u_{n}-x^{*}\right\|^{2}-\left\|u_{n}-u_{n+1}-\gamma \eta_{n} d_{n}\right\|^{2}-(2-\gamma) \gamma \eta_{n}^{2}\left\|d_{n}\right\|^{2} .
$$

Now, we estimate $\eta_{n}$. We have from (11) that

$$
\left\|d_{n}\right\| \leq\left\|u_{n}-v_{n}\right\|+\tau_{n}\left\|A u_{n}-A v_{n}\right\| \leq\left(1+\frac{\mu \tau_{n}}{\tau_{n+1}}\right)\left\|u_{n}-v_{n}\right\| .
$$

Hence

$$
\left\|d_{n}\right\|^{2} \leq\left(1+\frac{\mu \tau_{n}}{\tau_{n+1}}\right)^{2}\left\|u_{n}-v_{n}\right\|^{2}
$$

or equivalently

$$
\frac{1}{\left\|d_{n}\right\|^{2}} \geq \frac{1}{\left(1+\frac{\mu \tau_{n}}{\tau_{n+1}}\right)^{2}\left\|u_{n}-v_{n}\right\|^{2}}
$$

Again from (11) we find

$$
\begin{aligned}
\left\langle u_{n}-v_{n}, d_{n}\right\rangle & =\left\|u_{n}-v_{n}\right\|^{2}-\tau_{n}\left\langle u_{n}-v_{n}, A u_{n}-A v_{n}\right\rangle \\
& \geq\left\|u_{n}-v_{n}\right\|^{2}-\tau_{n}\left\|u_{n}-v_{n}\right\|\left\|A u_{n}-A v_{n}\right\| \\
& \geq\left\|u_{n}-v_{n}\right\|^{2}-\frac{\mu \tau_{n}}{\tau_{n+1}}\left\|u_{n}-v_{n}\right\|^{2} \\
& =\left(1-\frac{\mu \tau_{n}}{\tau_{n+1}}\right)\left\|u_{n}-v_{n}\right\|^{2} .
\end{aligned}
$$

Hence for all $n \geq n_{0}$

$$
\eta_{n}\left\|d_{n}\right\|^{2}=\left\langle u_{n}-v_{n}, d_{n}\right\rangle \geq\left(1-\frac{\mu \tau_{n}}{\tau_{n+1}}\right)\left\|u_{n}-v_{n}\right\|^{2}
$$

and

$$
\eta_{n}=\frac{\left\langle u_{n}-v_{n}, d_{n}\right\rangle}{\left\|d_{n}\right\|^{2}} \geq \frac{\left(1-\frac{\mu \tau_{n}}{\tau_{n+1}}\right)}{\left(1+\frac{\mu \tau_{n}}{\tau_{n+1}}\right)^{2}}
$$


Combining (23) and (24), we get

$$
\eta_{n}^{2}\left\|d_{n}\right\|^{2} \geq \frac{\left(1-\frac{\mu \tau_{n}}{\tau_{n+1}}\right)^{2}}{\left(1+\frac{\mu \tau_{n}}{\tau_{n+1}}\right)^{2}}\left\|u_{n}-v_{n}\right\|^{2} \quad \forall n \geq n_{0} .
$$

It follows from (21) and (25) that

$$
\left\|u_{n+1}-x^{*}\right\|^{2} \leq\left\|u_{n}-x^{*}\right\|^{2}-\left\|u_{n}-u_{n+1}-\gamma \eta_{n} d_{n}\right\|^{2}-(2-\gamma) \gamma \frac{\left(1-\frac{\mu \tau_{n}}{\tau_{n+1}}\right)^{2}}{\left(1+\frac{\mu \tau_{n}}{\tau_{n+1}}\right)^{2}}\left\|u_{n}-v_{n}\right\|^{2} \forall n \geq n_{0} .
$$

In the following result, we use the technique developed in [41, Lemma 3.3], where the sequential weak continuity of $A$ plays the key role, see also [42, 43].

Lemma 3.3. Assume that $A$ is Lipschitz continuous, pseudomonotone on $H$ and sequentially weakly continuous on $C$. If there exists a subsequence $\left\{u_{n_{k}}\right\}$ convergent weakly to $z \in H$ and $\lim _{k \rightarrow \infty}\left\|u_{n_{k}}-v_{n_{k}}\right\|=0$, then $z \in \Omega$.

Remark 3.1. The imposed sequential weak lower semicontinuity of $\|A x\|$ can be omitted in one of the following cases: either $A$ is monotone (see, [43]), or A is strongly pseudomonotone (see Theorem 3.2 below).

We are now in the position to establish the first main result of this section.

Theorem 3.1. Assume that $A$ is Lipschitz continuous, pseudomonotone on $H$ and sequentially weakly continuous on $C$. Then the sequence $\left\{u_{n}\right\}$ generated by Algorithm 3.1 converges weakly to an element of $\Omega$.

Proof: $\quad$ Let $p \in \Omega$. Thanks to Lemma 3.2 there exists $n_{0}>0$ such that

$$
\left\|u_{n+1}-x^{*}\right\|^{2} \leq\left\|u_{n}-x^{*}\right\|^{2}-\left\|u_{n}-u_{n+1}-\gamma \eta_{n} d_{n}\right\|^{2}-(2-\gamma) \gamma \frac{\left(1-\frac{\mu \tau_{n}}{\tau_{n+1}}\right)^{2}}{\left(1+\frac{\mu \tau_{n}}{\tau_{n+1}}\right)^{2}}\left\|u_{n}-v_{n}\right\|^{2} \quad \forall n \geq n_{0} .
$$

Thus

$$
\left\|u_{n+1}-x^{*}\right\| \leq\left\|u_{n}-x^{*}\right\| \quad \forall n \geq n_{0} .
$$

This implies that $\lim _{n \rightarrow \infty}\left\|u_{n}-x^{*}\right\|$ exists, thus the sequence $\left\{u_{n}\right\}$ is bounded. On the other hand, according to Lemma 3.2 , we get

$$
(2-\gamma) \gamma \frac{\left(1-\frac{\mu \tau_{n}}{\tau_{n+1}}\right)^{2}}{\left(1+\frac{\mu \tau_{n}}{\tau_{n+1}}\right)^{2}}\left\|u_{n}-v_{n}\right\|^{2} \leq\left\|u_{n}-x^{*}\right\|^{2}-\left\|u_{n+1}-x^{*}\right\|^{2} \quad \forall n \geq n_{0} .
$$

This implies that

$$
\lim _{n \rightarrow \infty}\left\|u_{n}-v_{n}\right\|=0
$$


Consequently, $\left\{v_{n}\right\}$ is bounded. Since $\left\{u_{n}\right\}$ is a bounded sequence, there exists the subsequence $\left\{u_{n_{k}}\right\}$ of $\left\{u_{n}\right\}$ such that $\left\{u_{n_{k}}\right\}$ converges weakly to $z \in H$. It follows from Lemma 3.3 and (26) that $z \in \Omega$.

Therefore, we have showed that:

i) For every $x^{*} \in \Omega$, then $\lim _{n \rightarrow \infty}\left\|u_{n}-x^{*}\right\|$ exists;

ii) Every sequential weak cluster point of the sequence $\left\{u_{n}\right\}$ is in $\Omega$.

By Lemma 2.2 the sequence $\left\{u_{n}\right\}$ converges weakly to an element of $\Omega$.

Remark 3.2. $\quad$ 1. Our result improves the related results in the literature and hence might be applied to a wider class of mappings. For example, we next present the advantage of our method compared with the recent result [14, Theorem 3.1]. In Theorem 3.1, $A: H \rightarrow H$ is assumed to be pseudomonotone on $H$ and sequentially weakly continuous on $C$ instead of monotone on $H$ in [14]. In particular, unlike [14, Algorithm 3.1] we use only one projection on the feasible set to design the proposed algorithm. Comparing with [39], our method does not require any line-search.

2. Similar to [4], since the sequence $\left(u_{n}\right)_{n \geq 0}$ generated by Algorithm 3.1 may not be feasible, we need to ask in the convergence analysis that $A$ is Lipschitz continuous on the whole space $H$. However, if the feasible set $C$ is bounded, then we can weaken this assumption by asking that $A$ is Lipschitz continuous on the bounded set

$$
D:=\{x+y: x \in C,\|y\| \leq d\},
$$

where d denotes the diameter of C (see [4, Remark 3.3]).

Before ending this section, we provide a result on the convergence rate of the iterative sequence generated by Algorithm 3.1.

Theorem 3.2. Assume that $A$ is L-Lipschitz continuous on $H$ and $\kappa$-strongly pseudomonotone on $C$. Then the sequence $\left\{u_{n}\right\}$ generated by Algorithm 3.1 converges strongly to the unique solution $x^{*}$ of (1) with a $Q$-linear rate.

Proof: Under assumptions made, it was proved that (1) has a unique solution [24]. Since $\left\langle A v_{n}, v_{n}-x^{*}\right\rangle \geq \kappa\left\|v_{n}-x^{*}\right\|^{2}$, from the $\kappa$-strong pseudomonotonicity of $A$, using (11) we have

$$
\begin{aligned}
\left\langle A u_{n}, x^{*}-v_{n}\right\rangle & =\left\langle A u_{n}-A v_{n}, x^{*}-v_{n}\right\rangle-\left\langle A v_{n}, v_{n}-x^{*}\right\rangle \\
& \leq\left\|A u_{n}-A v_{n}\right\|\left\|v_{n}-x^{*}\right\|-\kappa\left\|v_{n}-x^{*}\right\|^{2} \\
& \leq \frac{\mu}{\tau_{n+1}}\left\|u_{n}-v_{n}\right\|\left\|v_{n}-x^{*}\right\|-\kappa\left\|v_{n}-x^{*}\right\|^{2}
\end{aligned}
$$

By the definition of $v_{n}$ we have

$$
\left\langle u_{n}-\tau_{n} A u_{n}-v_{n}, v_{n}-x^{*}\right\rangle \geq 0 .
$$

Therefore

$$
\begin{aligned}
\left\langle u_{n}-v_{n}, x^{*}-v_{n}\right\rangle & \leq \tau_{n}\left\langle A u_{n}, x^{*}-v_{n}\right\rangle \\
& \leq \frac{\mu \tau_{n}}{\tau_{n+1}}\left\|u_{n}-v_{n}\right\|\left\|v_{n}-x^{*}\right\|-\tau_{n} \kappa\left\|v_{n}-x^{*}\right\|^{2}
\end{aligned}
$$


Thus

$$
\begin{aligned}
\tau_{n} \kappa\left\|v_{n}-x^{*}\right\|^{2} & \leq \frac{\mu \tau_{n}}{\tau_{n+1}}\left\|u_{n}-v_{n}\right\|\left\|v_{n}-x^{*}\right\|-\left\langle u_{n}-v_{n}, v_{n}-x^{*}\right\rangle \\
& \leq \frac{\mu \tau_{n}}{\tau_{n+1}}\left\|u_{n}-v_{n}\right\|\left\|v_{n}-x^{*}\right\|+\left\|u_{n}-v_{n}\right\|\left\|v_{n}-x^{*}\right\| \\
& =\left(1+\frac{\mu \tau_{n}}{\tau_{n+1}}\right)\left\|u_{n}-v_{n}\right\|\left\|v_{n}-x^{*}\right\| .
\end{aligned}
$$

This implies that

$$
\tau_{n} \kappa\left\|v_{n}-x^{*}\right\| \leq\left(1+\frac{\mu \tau_{n}}{\tau_{n+1}}\right)\left\|u_{n}-v_{n}\right\| .
$$

Since $\tau_{n} \geq \tau:=\min \left\{\tau_{0}, \frac{\mu}{L}\right\}$ for all $n \in \mathbb{N}$ and $\lim _{n \rightarrow \infty} \tau_{n}=\tau>0$, there exists $\beta>0$ such that $\tau \leq \tau_{n} \leq \beta$ for all $n$. Therefore, together with (27) we get

$$
\tau \kappa\left\|v_{n}-x^{*}\right\| \leq\left(1+\frac{\mu \beta}{\tau}\right)\left\|u_{n}-v_{n}\right\|=\frac{\tau+\mu \beta}{\tau}\left\|u_{n}-v_{n}\right\|
$$

Thus,

$$
\left\|v_{n}-x^{*}\right\| \leq \frac{\tau+\mu \beta}{\tau^{2} \kappa}\left\|u_{n}-v_{n}\right\|
$$

Moreover,

$$
\left\|u_{n}-x^{*}\right\| \leq\left\|u_{n}-v_{n}\right\|+\left\|v_{n}-x^{*}\right\| \leq\left(1+\frac{\tau+\mu \beta}{\tau^{2} \kappa}\right)\left\|u_{n}-v_{n}\right\| .
$$

This implies that

$$
\left\|u_{n}-v_{n}\right\| \geq \frac{\tau^{2} \kappa}{\tau^{2} \kappa+\tau+\mu \beta}\left\|u_{n}-x^{*}\right\| .
$$

From Lemma 3.2, there exists $n_{0}>0$ large enough such that

$$
\begin{gathered}
\left\|u_{n+1}-x^{*}\right\|^{2} \leq\left\|u_{n}-x^{*}\right\|^{2}-(2-\gamma) \gamma \frac{\left(1-\frac{\mu \tau_{n}}{\tau_{n+1}}\right)^{2}}{\left(1+\frac{\mu \tau_{n}}{\tau_{n+1}}\right)^{2}}\left\|u_{n}-v_{n}\right\|^{2} \quad \forall n \geq n_{0}, \\
1-\frac{\mu \tau_{n}}{\tau_{n+1}}>\frac{1-\mu}{2}>0 \quad \forall n \geq n_{0},
\end{gathered}
$$

and

$$
1+\frac{\mu \tau_{n}}{\tau_{n+1}} \leq 1+\frac{\mu \beta}{\tau}
$$

Combining (29), (30) and (31), we find that

$$
\left\|u_{n+1}-x^{*}\right\|^{2} \leq\left\|u_{n}-x^{*}\right\|^{2}-(2-\gamma) \gamma \frac{\left(\frac{1-\mu}{2}\right)^{2}}{\left(1+\frac{\mu \beta}{\tau}\right)^{2}}\left\|u_{n}-v_{n}\right\|^{2} \quad \forall n \geq n_{0} .
$$


Substituting (28) into (32), we get

$$
\begin{aligned}
\left\|u_{n+1}-x^{*}\right\|^{2} & \leq\left\|u_{n}-x^{*}\right\|^{2}-(2-\gamma) \gamma \frac{\left(\frac{1-\mu}{2}\right)^{2}}{\left(1+\frac{\mu \beta}{\tau}\right)^{2}}\left(\frac{\tau^{2} \kappa}{\tau^{2} \kappa+\tau+\mu \beta}\right)^{2}\left\|u_{n}-x^{*}\right\|^{2} \\
& =\left(1-(2-\gamma) \gamma \frac{\left(\frac{1-\mu}{2}\right)^{2}}{\left(1+\frac{\mu \beta}{\tau}\right)^{2}}\left(\frac{\tau^{2} \kappa}{\tau^{2} \kappa+\tau+\mu \beta}\right)^{2}\right)\left\|u_{n}-x^{*}\right\|^{2} \quad \forall n \geq n_{0} .
\end{aligned}
$$

Setting

$$
r:=1-(2-\gamma) \gamma \frac{\left(\frac{1-\mu}{2}\right)^{2}}{\left(1+\frac{\mu \beta}{\tau}\right)^{2}}\left(\frac{\tau^{2} \kappa}{\tau^{2} \kappa+\tau+\mu \beta}\right)^{2}
$$

we obtain

$$
\left\|u_{n+1}-x^{*}\right\|^{2} \leq r\left\|u_{n}-x^{*}\right\|^{2} \quad \forall n \geq n_{0},
$$

which implies that $r \geq 0$. Moreover, it is clear that $r<1$. Hence, the preceding inequality shows that $\left\{u_{n}\right\}$ converges linearly to $x^{*}$ with a Q-linear convergence rate $\sqrt{r} \in[0,1)$.

\section{Strong Convergence Analysis}

Although Theorem 3.2 provides the strong convergence of Algorithm 3.1 with a Q-linear rate, the restrictive condition that $A$ is strongly pseudomonotone prevents its applications. In this section, we incorporate the technique of Mann type method [29, 34] into Algorithm 3.1 to relax the condition strongly pseudomonotone and still obtain the strong convergence. The algorithm is of the form:

\section{Algorithm 4.1.}

Initialization: Given $\tau_{0}>0, \mu \in(0,1), \gamma \in(0,2)$. Let $u_{0} \in H$ be arbitrary

Iterative Steps: Given the current iterate $u_{n}$, calculate $u_{n+1}$ as follows:

Step 1. Compute

$$
v_{n}=P_{C}\left(u_{n}-\tau_{n} A u_{n}\right),
$$

If $u_{n}=v_{n}$ or $A v_{n}=0$ then stop and $v_{n}$ is a solution of $\Omega$. Otherwise

Step 2. Compute

$$
z_{n}=P_{T_{n}}\left(u_{n}-\gamma \tau_{n} \eta_{n} A v_{n}\right),
$$

where

$$
\begin{gathered}
T_{n}=\left\{x \in H \mid\left\langle u_{n}-\tau_{n} A u_{n}-v_{n}, x-v_{n}\right\rangle \leq 0\right\}, \\
\eta_{n}:= \begin{cases}\frac{\left\langle u_{n}-v_{n}, d_{n}\right\rangle}{\left\|d_{n}\right\|^{2}} & \text { if } d_{n} \neq 0, \\
0 & \text { if } d_{n}=0,\end{cases}
\end{gathered}
$$


and

$$
d_{n}:=u_{n}-v_{n}-\tau_{n}\left(A u_{n}-A v_{n}\right)
$$

Step 3. Compute

$$
u_{n+1}=\left(1-\alpha_{n}-\beta_{n}\right) u_{n}+\beta_{n} z_{n}
$$

update

$$
\tau_{n+1}:=\mu \frac{\left\|u_{n}-v_{n}\right\|}{\left\|A u_{n}-A v_{n}\right\|} \quad \text { if } \quad \tau_{n}\left\|A u_{n}-A v_{n}\right\|>\mu\left\|u_{n}-v_{n}\right\| \text {, otherwise } \tau_{n+1}:=\tau_{n} \text {. }
$$

Set $n:=n+1$ and go to Step 1 .

To guarantee the strong convergence, we assume that the sequences $\left\{\alpha_{n}\right\}$ and $\left\{\beta_{n}\right\}$ satisfy the following condition.

Condition 4.1. Let $\left\{\alpha_{n}\right\}$ and $\left\{\beta_{n}\right\}$ be two real sequences in $(0,1)$ such that $\left\{\beta_{n}\right\} \subset\left(a, 1-\alpha_{n}\right)$ for some $a>0$ and

$$
\lim _{n \rightarrow \infty} \alpha_{n}=0, \quad \sum_{n=1}^{\infty} \alpha_{n}=\infty .
$$

The main result of this section is established as follow:

Theorem 4.1. Assume that $A$ is Lipschitz continuous, pseudomonotone on $H$ and sequentially weakly continuous on $C$. Let $\left\{\alpha_{n}\right\}$ and $\left\{\beta_{n}\right\}$ satisfy Condition 4.1. Then the sequence $\left\{u_{n}\right\}$ generated by Algorithm 4.1 converges strongly to an element $x^{*} \in \Omega$, where $\left\|x^{*}\right\|=\arg \min \{\|z\|: z \in \Omega\}$.

Proof: First, we note from (13) that there always exists $n_{0} \in \mathbb{N}$ such that

$$
\left\|d_{n}\right\| \geq\left(1-\frac{\mu \tau_{n}}{\tau_{n+1}}\right)\left\|u_{n}-v_{n}\right\|>0 \quad \forall n \geq n_{0} .
$$

Furthermore, by Lemma 3.2, we have

$$
\left\|z_{n}-x^{*}\right\|^{2} \leq\left\|u_{n}-x^{*}\right\|^{2}-\left\|u_{n}-z_{n}-\gamma \eta_{n} d_{n}\right\|^{2}-(2-\gamma) \gamma \frac{\left(1-\frac{\mu \tau_{n}}{\tau_{n+1}}\right)^{2}}{\left(1+\frac{\mu \tau_{n}}{\tau_{n+1}}\right)^{2}}\left\|u_{n}-v_{n}\right\|^{2} \forall n \geq n_{0} .
$$

Thus

$$
\left\|z_{n}-x^{*}\right\| \leq\left\|u_{n}-x^{*}\right\| \quad \forall n \geq n_{0} .
$$

The proof will be divided into several steps.

Step 1. The sequence $\left\{u_{n}\right\}$ is bounded.

On the one hand, we have

$$
\begin{aligned}
\left\|u_{n+1}-x^{*}\right\| & =\left\|\left(1-\alpha_{n}-\beta_{n}\right) u_{n}+\beta_{n} z_{n}-x^{*}\right\| \\
& =\left\|\left(1-\alpha_{n}-\beta_{n}\right)\left(u_{n}-x^{*}\right)+\beta_{n}\left(z_{n}-x^{*}\right)-\alpha_{n} x^{*}\right\| \\
& \leq\left\|\left(1-\alpha_{n}-\beta_{n}\right)\left(u_{n}-x^{*}\right)+\beta_{n}\left(z_{n}-x^{*}\right)\right\|+\alpha_{n}\left\|x^{*}\right\| .
\end{aligned}
$$


On the other hand, from (36) we obtain that for all $n \geq n_{0}$

$$
\begin{aligned}
& \left\|\left(1-\alpha_{n}-\beta_{n}\right)\left(u_{n}-x^{*}\right)+\beta_{n}\left(z_{n}-x^{*}\right)\right\| \\
& \quad \leq\left(1-\alpha_{n}-\beta_{n}\right)\left\|u_{n}-x^{*}\right\|+\beta_{n}\left\|z_{n}-x^{*}\right\| \\
& \quad \leq\left(1-\alpha_{n}-\beta_{n}\right)\left\|u_{n}-x^{*}\right\|+\beta_{n}\left\|u_{n}-x^{*}\right\| \\
& \quad=\left(1-\alpha_{n}\right)\left\|u_{n}-x^{*}\right\|,
\end{aligned}
$$

which implies

$$
\left\|\left(1-\alpha_{n}-\beta_{n}\right)\left(u_{n}-x^{*}\right)+\beta_{n}\left(z_{n}-x^{*}\right)\right\| \leq\left(1-\alpha_{n}\right)\left\|u_{n}-x^{*}\right\| \quad \forall n \geq n_{0} .
$$

Combining (37) and (38), we deduce

$$
\begin{aligned}
\left\|u_{n+1}-x^{*}\right\| & \leq\left(1-\alpha_{n}\right)\left\|u_{n}-x^{*}\right\|+\alpha_{n}\left\|x^{*}\right\| \\
& \leq \max \left\{\left\|u_{n}-x^{*}\right\|,\left\|x^{*}\right\|\right\} \\
& \leq \ldots \leq \max \left\{\left\|u_{n_{0}}-x^{*}\right\|,\left\|x^{*}\right\|\right\}
\end{aligned}
$$

which means that the sequence $\left\{u_{n}\right\}$ is bounded and so is $\left\{z_{n}\right\}$.

Step 2. We prove that

$$
\begin{array}{r}
a(2-\gamma) \gamma \frac{\left(1-\frac{\mu \tau_{n}}{\tau_{n+1}}\right)^{2}}{\left(1+\frac{\mu \tau_{n}}{\tau_{n+1}}\right)^{2}}\left\|u_{n}-v_{n}\right\|^{2}+a\left\|u_{n}-z_{n}-\gamma \eta_{n} d_{n}\right\|^{2} \\
\quad \leq\left\|u_{n}-x^{*}\right\|^{2}-\left\|u_{n+1}-x^{*}\right\|^{2}+\alpha_{n}\left\|x^{*}\right\|^{2} \quad \forall n \geq n_{0} .
\end{array}
$$

Indeed, using (3) we have

$$
\begin{aligned}
\left\|u_{n+1}-x^{*}\right\|^{2}= & \left\|\left(1-\alpha_{n}-\beta_{n}\right) u_{n}+\beta_{n} z_{n}-x^{*}\right\|^{2} \\
= & \left\|\left(1-\alpha_{n}-\beta_{n}\right)\left(u_{n}-x^{*}\right)+\beta_{n}\left(z_{n}-x^{*}\right)+\alpha_{n}\left(-x^{*}\right)\right\|^{2} \\
= & \left(1-\alpha_{n}-\beta_{n}\right)\left\|u_{n}-x^{*}\right\|^{2}+\beta_{n}\left\|z_{n}-x^{*}\right\|^{2}+\alpha_{n}\left\|x^{*}\right\|^{2}-\beta_{n}\left(1-\alpha_{n}-\beta_{n}\right)\left\|u_{n}-z_{n}\right\|^{2} \\
& -\alpha_{n}\left(1-\alpha_{n}-\beta_{n}\right)\left\|u_{n}\right\|^{2}-\alpha_{n} \beta_{n}\left\|z_{n}\right\|^{2} \\
\leq & \left(1-\alpha_{n}-\beta_{n}\right)\left\|u_{n}-x^{*}\right\|^{2}+\beta_{n}\left\|z_{n}-x^{*}\right\|^{2}+\alpha_{n}\left\|x^{*}\right\|^{2} .
\end{aligned}
$$


It follows from (35) and (41) that for all $n \geq n_{0}$

$$
\begin{aligned}
\left\|u_{n+1}-x^{*}\right\|^{2} \leq & \left(1-\alpha_{n}-\beta_{n}\right)\left\|u_{n}-x^{*}\right\|^{2}+\beta_{n}\left\|u_{n}-x^{*}\right\|^{2}-\beta_{n}\left\|u_{n}-z_{n}-\gamma \eta_{n} d_{n}\right\|^{2} \\
& -\beta_{n}(2-\gamma) \gamma \frac{\left(1-\frac{\mu \tau_{n}}{\tau_{n+1}}\right)^{2}}{\left(1+\frac{\mu \tau_{n}}{\tau_{n+1}}\right)^{2}}\left\|u_{n}-v_{n}\right\|^{2}+\alpha_{n}\left\|x^{*}\right\|^{2} \\
= & \left(1-\alpha_{n}\right)\left\|u_{n}-x^{*}\right\|^{2}-\beta_{n}\left\|u_{n}-z_{n}-\gamma \eta_{n} d_{n}\right\|^{2} \\
& -\beta_{n}(2-\gamma) \gamma \frac{\left(1-\frac{\mu \tau_{n}}{\tau_{n+1}}\right)^{2}}{\left(1+\frac{\mu \tau_{n}}{\tau_{n+1}}\right)^{2}}\left\|u_{n}-v_{n}\right\|^{2}+\alpha_{n}\left\|x^{*}\right\|^{2} \\
\leq & \left\|u_{n}-x^{*}\right\|^{2}-\beta_{n}\left\|u_{n}-z_{n}-\gamma \eta_{n} d_{n}\right\|^{2} \\
& -\beta_{n}(2-\gamma) \gamma \frac{\left(1-\frac{\mu \tau_{n}}{\tau_{n+1}}\right)^{2}}{\left(1+\frac{\mu \tau_{n}}{\tau_{n+1}}\right)^{2}}\left\|u_{n}-v_{n}\right\|^{2}+\alpha_{n}\left\|x^{*}\right\|^{2} .
\end{aligned}
$$

Hence

$$
\begin{array}{r}
\beta_{n}(2-\gamma) \gamma \frac{\left(1-\frac{\mu \tau_{n}}{\tau_{n+1}}\right)^{2}}{\left(1+\frac{\mu \tau_{n}}{\tau_{n+1}}\right)^{2}}\left\|u_{n}-v_{n}\right\|^{2}+\beta_{n}\left\|u_{n}-z_{n}-\gamma \eta_{n} d_{n}\right\|^{2} \\
\quad \leq\left\|u_{n}-x^{*}\right\|^{2}-\left\|u_{n+1}-x^{*}\right\|^{2}+\alpha_{n}\left\|x^{*}\right\|^{2} \quad \forall n \geq n_{0} .
\end{array}
$$

Moreover, since $b_{n} \geq a$ for all $n$, we obtain

$$
\begin{array}{r}
a(2-\gamma) \gamma \frac{\left(1-\frac{\mu \tau_{n}}{\tau_{n+1}}\right)^{2}}{\left(1+\frac{\mu \tau_{n}}{\tau_{n+1}}\right)^{2}}\left\|u_{n}-v_{n}\right\|^{2}+a\left\|u_{n}-z_{n}-\gamma \eta_{n} d_{n}\right\|^{2} \\
\quad \leq\left\|u_{n}-x^{*}\right\|^{2}-\left\|u_{n+1}-x^{*}\right\|^{2}+\alpha_{n}\left\|x^{*}\right\|^{2} \quad \forall n \geq n_{0} .
\end{array}
$$

Step 3. We claim that

$$
\left\|u_{n+1}-x^{*}\right\|^{2} \leq\left(1-\alpha_{n}\right)\left\|u_{n}-x^{*}\right\|^{2}+\alpha_{n}\left[2 \beta_{n}\left\|u_{n}-z_{n}\right\|\left\|u_{n+1}-x^{*}\right\|+2\left\langle x^{*}, x^{*}-u_{n+1}\right\rangle\right] \quad \forall n \geq n_{0} .
$$

Indeed, setting $t_{n}=\left(1-\beta_{n}\right) u_{n}+\beta_{n} z_{n}$. We have for all $n \geq n_{0}$

$$
\begin{aligned}
\left\|t_{n}-x^{*}\right\| & =\left\|\left(1-\beta_{n}\right)\left(u_{n}-x^{*}\right)+\beta_{n}\left(z_{n}-x^{*}\right)\right\| \\
& =\left(1-\beta_{n}\right)\left\|u_{n}-x^{*}\right\|+\beta_{n}\left\|z_{n}-x^{*}\right\| \\
& \leq\left(1-\beta_{n}\right)\left\|u_{n}-x^{*}\right\|+\beta_{n}\left\|u_{n}-x^{*}\right\| \\
& =\left\|u_{n}-x^{*}\right\|,
\end{aligned}
$$

and

$$
\left\|t_{n}-u_{n}\right\|=\beta_{n}\left\|u_{n}-z_{n}\right\|
$$


Using (2), (43) and (44) we get for all $n \geq n_{0}$ that

$$
\begin{aligned}
\left\|u_{n+1}-x^{*}\right\|^{2} & =\left\|\left(1-\alpha_{n}-\beta_{n}\right) u_{n}+\beta_{n} z_{n}-x^{*}\right\|^{2} \\
& =\left\|\left(1-\beta_{n}\right) u_{n}+\beta_{n} z_{n}-\alpha_{n} u_{n}-x^{*}\right\|^{2} \\
& =\left\|\left(1-\alpha_{n}\right)\left(t_{n}-x^{*}\right)-\alpha_{n}\left(u_{n}-t_{n}\right)-\alpha_{n} x^{*}\right\|^{2} \\
& \leq\left(1-\alpha_{n}\right)^{2}\left\|t_{n}-x^{*}\right\|^{2}-2\left\langle\alpha_{n}\left(u_{n}-t_{n}\right)+\alpha_{n} x^{*}, u_{n+1}-x^{*}\right\rangle \\
& =\left(1-\alpha_{n}\right)^{2}\left\|t_{n}-x^{*}\right\|^{2}+2 \alpha_{n}\left\langle u_{n}-t_{n}, x^{*}-u_{n+1}\right\rangle+2 \alpha_{n}\left\langle x^{*}, x^{*}-u_{n+1}\right\rangle \\
& \leq\left(1-\alpha_{n}\right)\left\|t_{n}-x^{*}\right\|^{2}+2 \alpha_{n}\left\|u_{n}-t_{n}\right\|\left\|u_{n+1}-x^{*}\right\|+2 \alpha_{n}\left\langle x^{*}, x^{*}-u_{n+1}\right\rangle \\
& \leq\left(1-\alpha_{n}\right)\left\|u_{n}-x^{*}\right\|^{2}+\alpha_{n}\left[2 \beta_{n}\left\|u_{n}-z_{n}\right\|\left\|u_{n+1}-x^{*}\right\|+2\left\langle x^{*}, x^{*}-u_{n+1}\right\rangle\right] .
\end{aligned}
$$

Step 4. Finally, it remains to prove that $\left\{\left\|u_{n}-x^{*}\right\|\right\}$ converges to zero.

Indeed, by Lemma 2.4 it suffices to show that

$$
\limsup _{k \rightarrow \infty}\left(\beta_{n_{k}}\left\|u_{n_{k}}-z_{n_{k}}\right\|\left\|u_{n_{k}+1}-x^{*}\right\|+\left\langle x^{*}, x^{*}-u_{n_{k}+1}\right\rangle\right) \leq 0
$$

for every subsequence $\left\{\left\|u_{n_{k}}-x^{*}\right\|\right\}$ of $\left\{\left\|u_{n}-x^{*}\right\|\right\}$ satisfying

$$
\liminf _{k \rightarrow \infty}\left(\left\|u_{n_{k}+1}-x^{*}\right\|-\left\|u_{n_{k}}-x^{*}\right\|\right) \geq 0 .
$$

For this, suppose that $\left\{\left\|u_{n_{k}}-x^{*}\right\|\right\}$ is a subsequence of $\left\{\left\|u_{n}-x^{*}\right\|\right\}$ such that

$$
\liminf _{k \rightarrow \infty}\left(\left\|u_{n_{k}+1}-x^{*}\right\|-\left\|u_{n_{k}}-x^{*}\right\|\right) \geq 0 .
$$

Then

$\liminf _{k \rightarrow \infty}\left(\left\|u_{n_{k}+1}-x^{*}\right\|^{2}-\left\|u_{n_{k}}-x^{*}\right\|^{2}\right)=\liminf _{k \rightarrow \infty}\left[\left(\left\|u_{n_{k}+1}-x^{*}\right\|-\left\|u_{n_{k}}-x^{*}\right\|\right)\left(\left\|u_{n_{k}+1}-x^{*}\right\|+\left\|u_{n_{k}}-x^{*}\right\|\right)\right] \geq 0$.

By Step 2 we obtain

$$
\begin{aligned}
\limsup _{k \rightarrow \infty}\left(a(2-\gamma) \gamma \frac{\left(1-\frac{\mu \tau_{n_{k}}}{\tau_{n_{k}+1}}\right)^{2}}{\left(1+\frac{\mu \tau_{n_{k}}}{\tau_{n_{k}+1}}\right)^{2}}\left\|u_{n_{k}}-v_{n_{k}}\right\|^{2}\right. & \left.+a\left\|u_{n_{k}}-z_{n_{k}}-\gamma \eta_{n_{k}} d_{n_{k}}\right\|\right) \\
& \leq \limsup _{k \rightarrow \infty}\left[\left\|u_{n_{k}}-x^{*}\right\|^{2}-\left\|u_{n_{k}+1}-x^{*}\right\|^{2}+\alpha_{n_{k}}\left\|x^{*}\right\|^{2}\right] \\
& \leq \limsup _{k \rightarrow \infty}\left[\left\|u_{n_{k}}-x^{*}\right\|^{2}-\left\|u_{n_{k}+1}-x^{*}\right\|^{2}\right]+\limsup _{k \rightarrow \infty} \alpha_{n_{k}}\left\|x^{*}\right\|^{2} \\
& =-\liminf _{k \rightarrow \infty}\left[\left\|u_{n_{k}+1}-x^{*}\right\|^{2}-\left\|u_{n_{k}}-x^{*}\right\|^{2}\right] \\
& \leq 0 .
\end{aligned}
$$

This implies that

$$
\lim _{k \rightarrow \infty}\left\|u_{n_{k}}-v_{n_{k}}\right\|=0, \quad \lim _{k \rightarrow \infty}\left\|u_{n_{k}}-z_{n_{k}}-\gamma \eta_{n_{k}} d_{n_{k}}\right\|=0
$$

Now, we prove that

$$
\lim _{k \rightarrow \infty}\left\|u_{n_{k}+1}-u_{n_{k}}\right\|=0
$$


Indeed, we have

$$
0<\left(1-\frac{\mu \tau_{n}}{\tau_{n+1}}\right)\left\|u_{n}-v_{n}\right\| \leq\left\|d_{n}\right\| \leq\left(1+\frac{\mu \tau_{n}}{\tau_{n+1}}\right)\left\|u_{n}-v_{n}\right\| \quad \forall n \geq n_{0} .
$$

This implies that

$$
0<\frac{1}{\left(1+\frac{\mu \tau_{n}}{\tau_{n+1}}\right)^{2}\left\|u_{n}-v_{n}\right\|^{2}} \leq \frac{1}{\left\|d_{n}\right\|^{2}} \leq \frac{1}{\left(1-\frac{\mu \tau_{n}}{\tau_{n+1}}\right)^{2}\left\|u_{n}-v_{n}\right\|^{2}} \quad \forall n \geq n_{0} .
$$

Moreover from (22), (11) and the definition of $d_{n}$ we obtain

$$
0<\left(1-\frac{\mu \tau_{n}}{\tau_{n+1}}\right)\left\|u_{n}-v_{n}\right\|^{2} \leq\left\langle u_{n}-v_{n}, d_{n}\right\rangle \leq\left(1+\frac{\mu \tau_{n}}{\tau_{n+1}}\right)\left\|u_{n}-v_{n}\right\|^{2} \quad \forall n \geq n_{0} .
$$

From (48) and (49), we have

$$
0<\eta_{n}=\frac{\left\langle u_{n}-v_{n}, d_{n}\right\rangle}{\left\|d_{n}\right\|^{2}} \leq \frac{\left(1+\frac{\mu \tau_{n}}{\tau_{n+1}}\right)}{\left(1-\frac{\mu \tau_{n}}{\tau_{n+1}}\right)^{2}} \quad \forall n \geq n_{0} .
$$

It follows from (45) and (47) that

$$
\lim _{k \rightarrow \infty}\left\|d_{n_{k}}\right\|=0
$$

By (50) we get

$$
\eta_{n_{k}}\left\|d_{n_{k}}\right\| \leq \frac{\left(1+\frac{\mu \tau_{n_{k}}}{\tau_{n_{k}+1}}\right)}{\left(1-\frac{\mu \tau_{n_{k}}}{\tau_{n_{k}+1}}\right)^{2}}\left\|d_{n_{k}}\right\| \rightarrow 0 \text { as } k \rightarrow \infty
$$

Hence

$$
\left\|u_{n_{k}}-z_{n_{k}}\right\| \leq\left\|u_{n_{k}}-z_{n_{k}}-\gamma \eta_{n_{k}} d_{n_{k}}\right\|+\gamma \eta_{n_{k}}\left\|d_{n_{k}}\right\| \rightarrow 0 \text { as } k \rightarrow \infty
$$

Thus

$$
\left\|u_{n_{k}+1}-u_{n_{k}}\right\| \leq \alpha_{n_{k}}\left\|u_{n_{k}}\right\|+\beta_{n_{k}}\left\|u_{n_{k}}-z_{n_{k}}\right\| \rightarrow 0 \text { as } k \rightarrow \infty .
$$

Since the sequence $\left\{u_{n_{k}}\right\}$ is bounded, it follows that there exists a subsequence $\left\{u_{n_{k_{j}}}\right\}$ of $\left\{u_{n_{k}}\right\}$, which converges weakly to some $z \in H$, such that

$$
\limsup _{k \rightarrow \infty}\left\langle x^{*}, x^{*}-u_{n_{k}}\right\rangle=\lim _{j \rightarrow \infty}\left\langle x^{*}, x^{*}-u_{n_{k_{j}}}\right\rangle=\left\langle x^{*}, x^{*}-z\right\rangle .
$$

From $\lim _{k \rightarrow \infty}\left\|u_{n_{k}}-v_{n_{k}}\right\|=0$ and Lemma 3.3, we have $z \in \Omega$ and, from (51) and the definition of $x^{*}=P_{\Omega} 0$, we have

$$
\limsup _{k \rightarrow \infty}\left\langle x^{*}, x^{*}-u_{n_{k}}\right\rangle=\left\langle x^{*}, x^{*}-z\right\rangle \leq 0 .
$$

Combining (46) and (52), we have

$$
\begin{aligned}
\limsup _{k \rightarrow \infty}\left\langle x^{*}, x^{*}-u_{n_{k}+1}\right\rangle & \leq \limsup _{k \rightarrow \infty}\left\langle x^{*}, x^{*}-u_{n_{k}}\right\rangle \\
& =\left\langle x^{*}, x^{*}-z\right\rangle \leq 0 .
\end{aligned}
$$

Hence, by (53), $\lim _{k \rightarrow \infty}\left\|u_{n_{k}}-z_{n_{k}}\right\|=0$, Step 3 and Lemma 2.4, we have $\lim _{n \rightarrow \infty}\left\|u_{n}-x^{*}\right\|=0$. This is the desired result. 
Table 1: Averaged over 100 tests for fractional problems of different size

\begin{tabular}{|c|c|c|c|c|}
\hline $\mathrm{m}$ & $\begin{array}{c}\text { Algorithm } 3.1 \\
\text { Number of Iterations }\end{array}$ & time(sec.) & $\begin{array}{l}\text { Tseng's method [4] } \\
\text { Number of Iterations. }\end{array}$ & time \\
\hline 5 & 52.45 & 0.29 & 115.45 & 0.63 \\
\hline 10 & 52.27 & 0.28 & 120.43 & 0.64 \\
\hline 20 & 86.49 & 0.42 & 150.64 & 0.73 \\
\hline 50 & 106.80 & 0.52 & 161.69 & 0.80 \\
\hline 100 & 107.37 & 0.76 & 159.89 & 1.13 \\
\hline 200 & 107.58 & 0.81 & 164.51 & 1.24 \\
\hline
\end{tabular}

\section{$5 \quad$ Numerical Illustrations}

In this section, we present some numerical experiments to illustrate the performance of proposed Algorithms. As we are interested in pseudo-monotone VIs, in the first experiment we consider a class of pseudo-monotone VIs, which is not monotone. Consider the following quadratic fractional programming of the following form

$$
\min _{x \in C} f(x)
$$

where $f(x):=\frac{x^{T} M x+a^{T} x+c}{b^{T} x+d}$ and

$$
C=\left\{x \in \mathbb{R}^{m}: \sum_{i=1}^{m} x_{i} \leq m, 0 \leq x_{i} \leq 2 m, \quad i=1,2 \ldots, m\right\},
$$

The matrix $M$ is positive semi-definite and all elements are generated randomly in $(0,5)$. Similarly, vectors $a, b$ and scalars $c, d$ are generated randomly with elements in $(0,5)$. Clearly, this problem is equivalent to $\operatorname{VI}(A, C)$ with

$$
A x=\nabla f(x):=\frac{\left(b^{T} x+d\right)(2 M x+a)-b\left(x^{T} M x+a^{T} x+c\right)}{\left(b^{T} x+d\right)^{2}} .
$$

Since $f$ is pseudo-convex [5], $F$ is pseudo-monotone [20]. We compare Algorithm 3.1 with an adaptive version of Tseng's method [4]. We choose $\gamma=1$ for Algorithm 3.1, and the same parameters for both algorithms: $\tau_{0}=1, \mu=0.9$. All codes are implemented in Matlab $2019 b$ and we perform all computation on a MacBook Pro with 2.6 GHz Intel Core i7 and 16.00GB of memory. For each value of $m$, we perform 100 tests with random data and compare the average number of iterations and CPU time. The projections are computed using quadprog from Matlab. The stopping condition is $\left\|u_{n}-v_{n}\right\| \leq \epsilon=10^{-5}$. The results are displayed in Table 1. It can be seen that Algorithm 3.1 outperforms the Tseng's type method [4]. This is to be expected as Algorithm 3.1 uses the direction of projection contraction method, whose advantage was showed in $[6,21,38]$.

In the second experiment, we provide computational experiments illustrating the strong convergence method considered in Section 4 for solving VIs arising in optimal control problem. Let $0<T \in \mathbb{R}$, we denote by $L_{2}\left([0, T], \mathbb{R}^{m}\right)$ the Hilbert space of square integrable, measurable vector 
function $u:[0, T] \rightarrow \mathbb{R}^{m}$ with the inner product

$$
\langle u, v\rangle=\int_{0}^{T}\langle u(t), v(t)\rangle d t
$$

and norm

$$
\|u\|_{2}=\sqrt{\langle u, u\rangle}<\infty .
$$

We consider the following optimal control problem:

$$
u^{*}(t)=\operatorname{argmin}\{f(u): u \in U\}
$$

on the interval $[0, T]$, assuming that such a control exists. Here $U$ is the set of admissible controls, which has the form of an $m$-dimensional box and consists of piecewise continuous function:

$$
U=\left\{u(t) \in L_{2}\left([0, T], \mathbb{R}^{m}\right): u_{i}(t) \in\left[u_{i}^{-}, u_{i}^{+}\right], i=1,2, \ldots, m\right\} .
$$

Specially, the control can be bang-bang (piecewise constant function).

The terminal objective has the form

$$
f(u)=\phi(x(T)),
$$

where $\phi$ is a convex and differentiable function, defined on the attainability set.

Suppose that the trajectory $x(t) \in L_{2}([0, T]$ satisfies constrains in the form of a system of linear differential equation:

$$
\dot{x}(t)=D(t) x(t)+B(t) u(t), \quad x(0)=x_{0}, \quad t \in[0, T],
$$

where $D(t) \in \mathbb{R}^{n \times n}, B(t) \in \mathbb{R}^{n \times m}$ are given continuous matrices for every $t \in[0, T]$. By the Pontryagin maximum principle there exists a function $p^{*} \in L_{2}\left([0, T]\right.$ such that the triple $\left(x^{*}, p^{*}, u^{*}\right)$ solves for a.e. $t \in[0, T]$ the system

$$
\begin{aligned}
& \left\{\begin{array}{l}
\dot{x}^{*}(t)=D(t) x^{*}(t)+B(t) u^{*}(t) \\
x^{*}(0)=x_{0}
\end{array}\right. \\
& \left\{\begin{array}{c}
\dot{p}^{*}(t)=-D(t)^{\top} p^{*}(t) \\
p^{*}(T)=\nabla g(x(T))
\end{array}\right. \\
& 0 \in B(t)^{\top} p^{*}(t)+N_{U}\left(u^{*}(t)\right),
\end{aligned}
$$

where $N_{U}(u)$ is the normal cone to $U$ at $u$ defined by

$$
N_{U}(u):= \begin{cases}\emptyset & \text { if } u \notin U, \\ \{\ell \in H:\langle\ell, v-u\rangle \leq 0, \forall v \in U\} & \text { if } u \in U .\end{cases}
$$

Denoting $G u(t):=B(t)^{\top} p(t)$, it is known that $G u$ is the gradient of the objective cost function $f$ [30]. We can write (54) as the following monotone variational inequality

$$
\left\langle G u^{*}, v-u^{*}\right\rangle \geq 0 \quad \forall v \in U .
$$

The following example is the control of a harmonic oscillator taken from [33, Example 7].

$$
\begin{array}{ll}
\operatorname{minimize} & x_{2}(3 \pi) \\
\text { subject to } & \dot{x_{1}}(t)=x_{2}(t), \\
& \dot{x_{2}}(t)=-x_{1}(t)+u(t), \forall t \in[0,3 \pi], \\
& x(0)=0, \\
& u(t) \in[-1,1] .
\end{array}
$$


The exact optimal control in this problem is known:

$$
u^{*}(t)=\left\{\begin{aligned}
1 & \text { if } t \in[0, \pi / 2) \cup(3 \pi / 2,5 \pi / 2), \\
-1 & \text { if } t \in(\pi / 2,3 \pi / 2) \cup(5 \pi / 2,3 \pi] .
\end{aligned}\right.
$$

We choose the following parameters for Algorithm 4.1:

$$
\tau_{0}=1, \mu=0.95, \gamma=1, \alpha_{n}=10^{-4} /(n+1), \beta_{n}=0.95-\alpha_{n} .
$$

The initial control $u_{0}(t)$ is chosen randomly in $[-1,1]$, and the stopping condition is Error $=\| u_{n+1}-$ $u_{n} \| \leq \epsilon=10^{-5}$. The approximate solution is obtained after 102 iterations in 0.095288 seconds of CPU time as shown in Figure 1.


Figure 1: Random initial control (green) and optimal control (red) on the left and optimal trajectories on the right for the control of a harmonic oscillator computed by Algorithm 4.1.

In Figure 2 compares the performance of Algorithm 4.1 with three other strong convergence algorithms: [28]-denoted by KS-Method, [36, Algorithm 3.1]- denoted by SI-Method and [43, Algorithm 3.3]- denoted by SV-Method. For the KS-Method, we choose adpative stepsize $\lambda_{n+1}=$ $\min \left\{\lambda_{n}, \frac{\mu\left\|y_{n}-x_{n}\right\|}{\left\|A y_{n}-A x_{n}\right\|}\right\}$ since the Lipschitz constant of $A$ is not available. For [36, Algorithm 3.1] we choose $\mu=0.5, \rho=0.5$ and for [43, Algorithm 3.3] we choose $\gamma=0.5, \rho=0.5$ as used in these papers. It can be seen that Algorithm 4.1 is takes advantage comparing with the other methods.

\section{Conclusions}

In this paper we presented some improved results of the subgradient extragradient method for solving pseudomonotone variational inequalities in real Hilbert spaces. The algorithms require the calculation of only one projection onto the feasible set $C$ per iteration. Using an adaptive stepsize rule, the convergence of the proposed algorithms does not require knowledge of the Lipschitz constant of $A$ in priori. Numerical experiments for fractional programming and optimal control problems are presented to illustrate the performance of the new methods. 




Figure 2: Comparision Algorithm 4.1 with three other strong convergence algorithms

\section{Acknowledgements}

The authors are very thankful to both referees for their careful reading and contructive comments, which helped improving the presentation of the paper. This work was supported by the Vietnam National Foundation for Science and Technology Development (NAFOSTED) project 101.01-2019.320. A significant part of the paper was completed during a scientific stay of the authors at the Vietnam Institute for Advanced Study in Mathematics (VIASM), whose support and hospitality are gratefully appreciated.

\section{References}

1. Antipin, A.S.: On a method for convex programs using a symmetrical modification of the Lagrange function. Ekonomika i Mat. Metody. 12, 1164-1173 (1976)

2. Avriel, M., Diewert, W. E., Schaible, S., Zang, I.: Generalized Concavity. Society for Industrial and Applied Mathematics (2010)

3. Baiocchi, C., Capelo, A.: Variational and Quasivariational Inequalities, Applications to Free Boundary Problems. Wiley, New York (1984)

4. Bot,, R.I., Csetnek, E.R., Vuong, P.T.: The forward-backward-forward method from continuous and discrete perspective for pseudo-monotone variational inequalities in Hilbert spaces. Eur. J. Oper. Res. 287, 49-60 (2020) 
5. Borwein, J. M., Lewis, A.S.: Convex Analysis and Nonlinear Optimization: Theory and Examples. Springer Science and Business Media, New York (2006)

6. Cai, X., Gu, G., He, B.: On the $O(1 / t)$ convergence rate of the projection and contraction methods for variational inequalities with Lipschitz continuous monotone operators. Comput. Optim. Appl. 57 (2014), 339-363.

7. Cegielski, A.: Iterative Methods for Fixed Point Problems in Hilbert Spaces. Lecture Notes in Mathematics, vol. 2057. Springer, Berlin (2012)

8. Ceng, L. C., Teboulle, M, Yao, J. C.: Weak convergence of an iterative method for pseudomonotone variational inequalities and fixed-point problems. J. Optim. Theory Appl. 146, 19-31, (2010)

9. Censor, Y., Gibali A, Reich S.: The subgradient extragradient method for solving variational inequalities in Hilbert space. J. Optim. Theory Appl. 148, 318-335 (2011)

10. Censor, Y., Gibali, A., Reich, S.: Strong convergence of subgradient extragradient methods for the variational inequality problem in Hilbert space. Optim. Meth. Softw. 26, 827-845 (2011)

11. Censor, Y., Gibali, A., Reich, S.: Extensions of Korpelevich's extragradient method for the variational inequality problem in Euclidean space. Optimization. 61, 1119-1132 (2011)

12. Cottle, R.W., Yao, J.C.: Pseudo-monotone complementarity problems in Hilbert space. J. Optim. Theory Appl. 75, 281-295 (1992)

13. Dong, L.Q., Cho, J.Y., Zhong, L.L., Rassias, M.Th.: Inertial projection and contraction algorithms for variational inequalities. J. Glob. Optim. 70, 687-704 (2018)

14. Dong, Q.L., Gibali, A., Jiang, D.: A modified subgradient extragradient method for solving the variational inequality problem. Numer. Algorithms. 79, 927-940 (2018)

15. Gibali, A., Reich, S., Zalas, R: Iterative methods for solving variational inequalities in Euclidean space. J. Fixed Point Theory Appl. 17, 775-811, (2015)

16. Gibali, A., Reich, S., Zalas, R: Outer approximation methods for solving variational inequalities in Hilbert space. Optimization. 66, 417-437 (2017)

17. Facchinei, F., Pang, J.S.: Finite-Dimensional Variational Inequalities and Complementarity Problems. Springer Series in Operations Research, vols. I and II. Springer, New York (2003)

18. Fichera, G.: Sul problema elastostatico di Signorini con ambigue condizioni al contorno. Atti Accad. Naz. Lincei, VIII. Ser., Rend., Cl. Sci. Fis. Mat. Nat. 34 (1963), 138-142.

19. Goebel, K., Reich, S.: Uniform Convexity, Hyperbolic Geometry, and Nonexpansive Mappings. Marcel Dekker, New York (1984)

20. Hadjisavvas, N., Komlosi, S., Schaible, S. (eds.): Handbook on Generalized Convexity and Generalized Monotonicity, Nonconvex optimization and its Applications, vol. 76. Springer, New York (2005).

21. He, B.S.: A class of projection and contraction methods for monotone variational inequalities. Appl. Math. Optim. 35, 69-76 (1997) 
22. Karamardian, S., Schaible, S.: Seven kinds of monotone maps. J. Optim. Theory Appl. 66, 37-46 (1990)

23. Khanh, P.D.: A modified extragradient method for infinite-dimensional variational inequalities. Acta. Math. Vietnam. 41, 251-263 (2016)

24. Kim, D. S., Vuong, P. T., Khanh P. D.: Qualitative properties of strongly pseudomonotone variational inequalities. Optim. Lett. 10, 1669-1679 (2016)

25. Kinderlehrer, D., Stampacchia, G.: An introduction to variational inequalities and their applications. Academic, New York 1980.

26. Konnov, I. V.: Combined Relaxation Methods for Variational Inequalities. Springer-Verlag, Berlin (2001)

27. Korpelevich, G.M.: The extragradient method for finding saddle points and other problems. Ekonomika i Mat. Metody. 12, 747-756 (1976)

28. Kraikaew, R., Saejung, S.: Strong convergence of the Halpern subgradient extragradient method for solving variational inequalities in Hilbert spaces. J. Optim. Theory Appl. 163, 399-412 (2014)

29. Mann, W.R.: Mean value methods in iteration. Proc. Amer. Math. Soc. 4, 506-510 (1953)

30. Nikol'skii, M.S.: Convergence of the gradient projection method in optimal control problems. Comp. Math. Model. 18, 148-156 (2007)

31. Opial, Z.: Weak convergence of the sequence of successive approximations for nonexpansive mappings. Bull. Amer. Math. Soc. 73, 591-597 (1967)

32. Ortega, J.M., Rheinboldt, W.C.: Iterative Solution of Nonlinear Equations in Several Variables. Academic Press, New York (1970)

33. Pietrus, A., Scarinci, T., Veliov, V.M.: High order discrete approximations to Mayer's problems for linear systems. SIAM J. Control Optim. 56, 102-119 (2018)

34. Reich, S.: Weak convergence theorems for nonexpansive mappings in Banach spaces. J. Math. Anal. Appl. 24, 269-272 (1978)

35. Saejung, S., Yotkaew, P.: Approximation of zeros of inverse strongly monotone operators in Banach spaces. Nonlinear Anal. 75, 742-750 (2012)

36. Shehu, Y., Iyiola, O.S.: Strong convergence result for monotone variational inequalities. Numer. Algorithms, 76, 259-282 (2017)

37. Stampacchia, G.: Formes bilineaires coercitives sur les ensembles convexes. C. R. Acad. Sci., 258 (1964), 4413-4416

38. Sun, D.F.: A class of iterative methods for solving nonlinear projection equations. J. Optim. Theory Appl. 91, 123-140 (1996)

39. Thong, D.V., Shehu, Y., Iyiola O.S.: Weak and strong convergence theorems for solving pseudomonotone variational inequalities with non-Lipschitz mappings. Numer. Algorithms. 84, 795$823(2020)$ 
40. Thong, D.V., Hieu, D.V.: Modified subgradient extragradient method for variational inequality problems. Numer. Algorithms. 79, 597-610 (2018)

41. Thong, D.V., Vuong, P.T.: Modified Tseng's extragradient methods for solving pseudomonotone variational inequalities. Optimization. 68, 2207-2226 (2019)

42. Vuong, P.T.: On the weak convergence of the extragradient method for solving pseudomonotone variational inequalities. J. Optim. Theory Appl. 176, 399-409 (2018)

43. Vuong P.T., Shehu, Y.: Convergence of an extragradient-type method for variational inequality with applications to optimal control problems. Numer. Algorithms. 81, 269-291(2019)

44. Yang, J., Liu, H., Liu, Z.: Modified subgradient extragradient algorithms for solving monotone variational inequalities. Optimization. 67, 2247-2258(2018) 